\title{
Problem of Materials for Electromagnetic Launchers
}

\author{
Gennady Shvetsov and Sergey Stankevich \\ Lavrentyev Institute of Hydrodynamics Novosibirsk \\ Russia
}

\section{Introduction}

During the last twenty years, considerable attention of researchers working in the areas of pulsed power, plasma physics, and high-velocity acceleration of solids has been given to electromagnetic methods of accelerating solids. These issues were the subject of more than twenty international conferences in the U.S. and European countries. Papers on this topic occupy an important place in the programs of international conferences on pulsed power, plasma physics, megagauss magnetic field generation, etc. The increased interest of the world scientific community in problems of electromagnetic acceleration of solids to high velocities is due to the high scientific and practical importance of high-velocity impact research. Accelerators of solids are used to study the equations of state for solids under extreme conditions, simulate the effects of meteorite impact on spacecraft, investigate problems related to missile defense, test various artillery systems and weapons, etc.

Information on the development and current status of research on electromagnetic methods for high-velocity acceleration of solids in the United States, Russia, France, Germany, Greate Britain, China and other country can be found in reviews (Fair, 2005, 2007; Shvetsov et al., 2001, 2003, 2007; Lehmann, 2003; Haugh \& Gilbert, 2003; Wang, 2003).

For high-velocity accelerators of solids, the most important are two characteristics and answers to the following two questions: 1) what absolute velocities can be achieved in a particular type of launcher for a body of a given mass? and 2) what is the service life of the launcher? An analysis of existing theoretical concepts and available experimental data has shown that the most severe limitations in attaining high velocities and providing acceptable service life of electromagnetic launchers are thermal limitations due to the circuit current. A number of crisis (critical) phenomena and processes have been found that disrupt the normal mode of accelerator operation and lead to the destruction of the accelerated body or accelerator or to the termination of the acceleration.

In electromagnetic plasma armature railguns, one of the main factors limiting the projectile velocity is the erosion of rails and insulators, leading to an increase in the mass accelerated in the launchers, an increase in the density of the gas moving in the channel, an increase in viscous friction, and a decrease in the dielectric strength of the railgun channel, which can cause a secondary breakdown in the channel with the formation of a new arc and setting an additional mass of gas in motion, etc. The main factor responsible for the intense erosion of materials is their heating by the radiation from the plasma armature to temperatures above the melting and vaporization temperatures of the materials. 
In coil guns, Joule heating by the current results in a reduction in the mechanical strength of projectiles up to its complete loss during melting. Magnetic forces can lead to deformation and fracture of the inductor and accelerated body and other phenomena.

The main problem limiting the attainment of high velocities in metal armature railguns is the problem of preserving the sliding metallic contact at high velocities. An increase in the current density near the rear surface of the armature, due mainly to the velocity skin effect, leads to rapid heating, melting, and vaporization of the armature near the contact boundary. The development of these processes result in a rapid transition to an arc contact mode, enhancement of erosion processes, reduction or termination of the acceleration, and destruction of the barrel and accelerated body (Barber et al., 2003).

One of the necessary conditions for the implementation of crisis-free acceleration is the requirement that the elements of the launcher and accelerated body be heated below the melting point throughout the acceleration. The heating limitation condition implies restrictions on the maximum value of the magnetic field strength and the maximum linear current density in electromagnetic launchers and to a limitation on the velocity.

In the chapter, the velocity to which a solid of a given mass can be accelerated at a certain distance provided that, during acceleration, the temperature of the rails and accelerated body does not exceed certain values critical for the type of launcher and material used is considered the ultimate velocity in terms of the heating conditions or simply the ultimate velocity.

An analysis has shown that the ultimate velocities can be substantially increased by using composite conductors with controllable thermal properties and by optimizing the shape of the current pulse. Thus, the problem of materials and thermal limitations for electromagnetic launchers of solids is central to the study of their potential.

This chapter presents the results of studies of thermal limitations in attaining high velocities in electromagnetic launchers; analyzes the possibility of increasing the ultimate (in terms of heating conditions) velocities of accelerated solids in subcritical modes of operation of electromagnetic launchers of various types (plasma armature railguns, induction and rail accelerators of conducting solids) taking into account the limitations imposed on the heating of the launcher and accelerated body during acceleration; and investigates various ways to increase the ultimate kinematic characteristics of launchers through the use of composite conductors of various structures and with various electrothermal properties as currentcarrying elements.

\section{Problems of materials in plasma-armature railguns}

In analyzing various physical factors that limit the performance of plasma-armature railguns, it is convenient to use the concept of the critical current density $I^{*} / b\left(I^{*}\right.$ is the current in the circuit, $b$ is the width of the electrodes) above which these factors begin to manifest themselves. This was apparently first noted in (Barber, 1972). Estimates show (Barber, 1972; Shvetsov et al., 1987) that the smallest value of $I^{*} / b$ is obtained from the condition that the current flowing in the circuit must not lead to melting of the electrodes and, consequently, to high erosion.

Investigation of the ultimate capabilities of the erosion-free operation of plasma-armature railgun requires, first of all, knowledge of the plasma armature, railgun, and power supply characteristics necessary for this operation regime. As shown by experimental studies (Hawke \& Scudder, 1980; Shvetsov et al., 1987), plasma-armature properties (length $l_{\mathrm{p}}$, 
average density $\rho_{\mathrm{p}}$, impedance $r_{\mathrm{p}}$ ) differ only slightly from some typical values $r_{\mathrm{p}} \sim 1$ mohm, $l_{\mathrm{p}} \sim(5 \div 10) \cdot b$, and $\rho_{\mathrm{p}} \sim 10 \div 30 \mathrm{~kg} / \mathrm{m}^{3}$ in both different experimental setups and in the acceleration process. Thus, for a given accelerator channel cross section and a given projectile mass, the only parameters that can be varied to control the development or slowing of erosion processes are the linear current density in the accelerator $I / b$ and the thermophysical properties of the electrode material.

In analysis of the possibilities of increasing $I^{*} / b$, the question naturally arises as to whether composite materials can be used for this purpose. Prerequisites for increasing $I^{*} / b$ are the well-known fact of high erosion resistance of composite materials in highcurrent switches (in 1.5-3 times higher the resistance of tungsten) and the assumption that in a railgun, the plasma armature interacts with the electrodes in the same way as in highcurrent switches (Jackson et al., 1986). A number of papers have reported experiments with electrodes coated with high-melting materials such as $\mathrm{W}-\mathrm{Cu}, \mathrm{W} / \mathrm{Re}-\mathrm{Cu}, \mathrm{Mo}-\mathrm{Cu}$, etc. (Harding et al., 1986; Shrader et al., 1986; Vrabel et al., 1991; Shvetsov, Anisimov et al., 1992). It has been noted that the coated copper electrodes $(\mathrm{W}-\mathrm{Cu}, \mathrm{W} / \mathrm{Re}-\mathrm{Cu})$ offer advantages over uncoated ones for use in rail launchers under the same conditions.

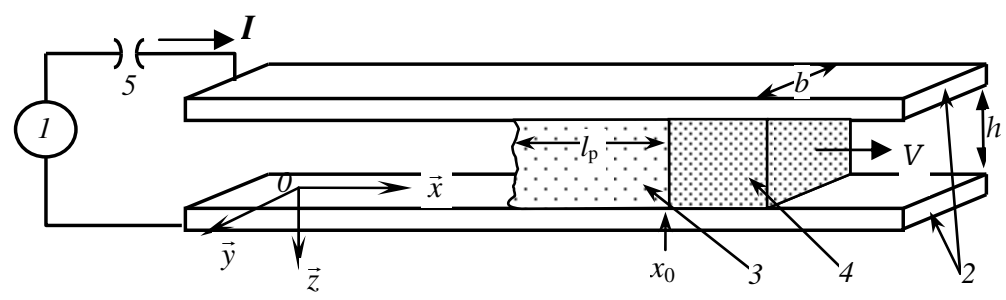

Fig. 1. Schematic diagram of the plasma-armature launcher of solids. 1 - power supply, 2 electrodes, 3 - plasma armature, 4 -projectile, 5 - switch.

We will analyze the possibilities of increasing the critical current density by using composite electrodes in conventional plasma-armature railguns. A schematic diagram of a plasma armature launcher of dielectric solids is shown in Figure 1, where 1 is the current source, 2 are electrodes, 3 is the plasma armature, 4 is the projectile, 5 is the switch, $l_{\mathrm{p}}$ is the length of the plasma armature, $b$ is the electrode width, and $h$ is the distance between the electrodes. When the switch 5 is closed, a current starts to flow in the circuit, producing an electromagnetic force which accelerates the plasma armature and the projectile.

We will assume that changes in the electrode temperature are only due to the effect of the heat flux from the plasma. As shown in (Shvetsov et al. 1987), if the temperature change due to Joule heating is neglected, the error in determining the surface temperature is usually not more than a few percents. The problem of determining the temperature in some local neighborhood of a point $x_{0}$ on the electrode surface can be regarded as the problem of heating of a half-space $z>0$ which generally has inhomogeneous thermophysical properties by a heat flux $q$ acting for a time $\Delta t\left(x_{0}\right)$ equal to the time during which the plasma armature passes over the point $x_{0}$. This problem reduces to solving the heat-conduction equation with a given initial temperature distribution and boundary conditions:

$$
\rho c \frac{\partial T}{\partial t}=\operatorname{div}(k \operatorname{grad} T)
$$




$$
\begin{gathered}
T\left(x, y, z, t \leq t_{0}\left(x_{0}\right)\right)=T_{0} \\
-\left.k \frac{\partial T}{\partial z}\right|_{z=0}=q(t),\left.T\right|_{z \rightarrow \infty}=T_{0}, t_{0}\left(x_{0}\right) \leq t \leq t_{0}\left(x_{0}\right)+\Delta t_{0}\left(x_{0}\right)
\end{gathered}
$$

where in the general case density $\rho$, heat capacity $c$, and thermal conductivity $k$ may be functions of $x, y$, and $z$ depending on temperature $T$. $T_{0}$. is the initial temperature, $t_{0}\left(x_{0}\right)$ is the time of arrival of plasma armature to the point $x_{0}$.

Following (Powell, 1984; Shvetsov et al., 1987) let us consider that the armature moves as a solid body with constant mass, length $l$, and electric resistance $r$. Neglect the variation in the internal thermal energy of plasma armature and assume that all energy dissipating in it uniformly releases through the surface limiting the volume occupied by plasma. In this case, all released energy is absorbed in the channel of the railgun as if the release had happened in a vacuum.

These assumptions make it possible to establish a simple connection between the total current $I$ through plasma armature and the intensity of heat flux $q$ from its surface:

$$
q=\frac{r_{\mathrm{p}} I^{2}}{S},
$$

where $S$ is the area of plasma armature surface.

The dynamics of plasma armature and the projectile is determined by integrating the equations of motion:

$$
\frac{d V}{d t}=\frac{\lambda}{2 m} I^{2}, \frac{d L}{d t}=V
$$

where $\lambda$ is the inductance per unit-length of railgun channel, $m$ is the sum of mass of plasma and projectile, and $V$ is projectile velocity.

The critical current density is determined under the condition that the temperature at any point $x_{0}, y$ on the electrode surface $(z=0)$ during the acceleration does not exceed the critical temperature $T^{*}$ of the electrode material (the melting temperature for homogeneous materials or the melting or evaporation temperatures for one of the components of composite material).

Dependences of the current density on time or the distance $L$ traveled by the plasma armature can be obtained by simultaneously solving equations (1)-(3).

A similarity analysis for the thermal problem (1) shows that the maximum temperature $T_{\max }(K)$ of a homogeneous electrode and a composite electrode consisting of a mixture of fairly small particles depends only on the magnitude of the thermal action $K=q \sqrt{\Delta t}$. The magnitude of the thermal action $K^{*}$ at which the electrode surface reaches the critical temperature $T_{\max }\left(K^{*}\right)=T^{*}$ depends only on the thermophysical properties of the electrode material and can be regarded as a characteristic of the heat resistance of the material heated by a pulsed heat flux (Shvetsov \& Stankevich, 1995).

The maximum projectile velocity in plasma-armature railguns subject to the electrode heating constraint is achieved when the shape of the current pulse provides a constant thermal action at each point of the electrode surface and the magnitude of this action is 
equal to the heat resistance of the electrode material. It is established that the dependences of the critical current density and the ultimate projectile velocity on the traveled distance $I^{*}(L) / b, V(L)$ for a railgun accelerator with electrodes made of an arbitrary material $X$ are linked to the corresponding dependences for the same accelerator with copper electrodes by the relations

$$
\left(\frac{I^{*}(L)}{b}\right)_{X}=\alpha\left(\frac{I^{*}(L)}{b}\right)_{\mathrm{Cu}}, \quad V_{X}(L)=\alpha V_{\mathrm{Cu}}(L)
$$

where the coefficient $\alpha=\alpha_{X} / \alpha_{\mathrm{Cu}}=\left(K_{X}^{*} / K_{\mathrm{Cu}}^{*}\right)^{2 / 3}$ characterizes the relative heat resistance of the material $X$ with respect to the heat resistance of copper.

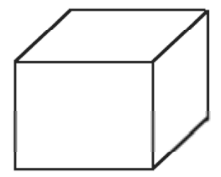

$a$

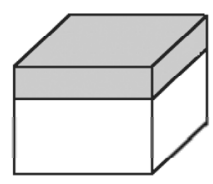

$b$

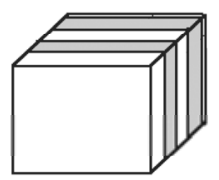

$c$

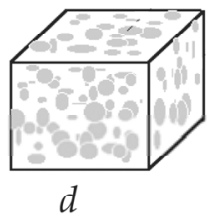

d

Fig. 2. Electrode structures. $a$ ) homogeneous electrode, $b$ ) coated electrode, $c$ ) multilayer electrode with vertical layers, $d$ ) composite electrode consisting of a mixture of powders.

\begin{tabular}{|c|c|c|c|c|c|c|c|c|}
\hline $\mathrm{Cu}$ & $\mathrm{Mo}$ & $\mathrm{W}$ & $\mathrm{Al}$ & $\mathrm{Ta}$ & $\mathrm{Re}$ & $\mathrm{Cr}$ & $\mathrm{Fe}$ & $\mathrm{Ni}$ \\
\hline 1.0 & 1.17 & 1.38 & 0.55 & 0.99 & 0.99 & 0.87 & 0.69 & 0.78 \\
\hline
\end{tabular}

Table 1. Homogeneous metals

An analysis was made of the heat resistance and critical current density for electrodes of various structures: a homogeneous electrode (Fig. 2, a), an electrode with a high-melting coating (Fig. 2, b), an electrode with vertical layers of different metals (Fig. 2, c), and a composite electrode consisting of a mixture of particles (Fig. 2, d).

Calculations of the coefficient of relative heat resistance of homogeneous electrodes of metals such as $\mathrm{W}, \mathrm{Mo}, \mathrm{Re}, \mathrm{Ta}, \mathrm{Cr}, \mathrm{Ni}, \mathrm{Fe}$, and $\mathrm{Al}$ showed that only tungsten and molybdenum electrodes can compete with copper $\left(\alpha_{\mathrm{W}}=1.38, \alpha_{\mathrm{Mo}}=1.17\right)$, and for other metals $\alpha<1$ (Table 1 ).

An increase in the heat resistance of electrodes coated with a high-melting material (Fig. 2, b) is possible if the thermal conductivity of the base material is higher than the coating thermal conductivity and the heating rate of the base at a given heat flux is lower than that of the coating. The maximum increase in heat resistance is achieved at an optimal coating thickness at which the temperatures of the surface and the interface between the materials simultaneously reach the values critical to the coating and base materials. The optimum coating thickness depends on the heat flux and the duration of heat pulse; therefore, to maintain the highest possible linear current density for a given pair of materials, the coating thickness along the electrode should decrease according to a definite law. The results of calculations of the relative heat resistance of copper electrodes coated with various metals are presented in Table 2 . 


\begin{tabular}{|c|c|c|c|c|c|c|}
\hline & W-Cu & Ta-Cu & Mo-Cu & Re-Cu & Cr-Cu & Os-Cu \\
\hline$\alpha_{1}$ & 1.443 & 1.188 & 1.299 & 1.197 & 1.124 & 1.312 \\
\hline$\alpha_{2}$ & 1.628 & 1.358 & 1.445 & 1.344 & 1.202 & 1.487 \\
\hline
\end{tabular}

Table 2. Coated electrodes

The calculations were performed for two cases: in the first, it was assumed that during the time of passage of the plasma armature, both materials remain in the solid phase $\left(\alpha_{1}\right)$, and in the second case, melting of the base to a depth equal to the coating thickness $\left(\alpha_{2}\right)$ was allowed. One can see that with the use of copper electrodes with an optimized thickness of the tungsten coating, the heat resistance coefficient (and the maximum velocity) increases to a value $\alpha_{\mathrm{W} / \mathrm{Cu}}=1.45$ under the maximum heating to the melting temperature, and to a value $\alpha_{\mathrm{W} / \mathrm{Cu}}=1.68$ in the case where during the travel time of the thermal pulse, the copper base is melted to a depth equal to the coating thickness and the surface tungsten layer remains in the solid phase.

Analysis of the problem of heating of electrodes with vertical layers (Fig. 2,c) and composite electrodes consisting of a mixture of particles (Fig. 2, $d$ ) by a heat flux pulse shows that for electrodes of this type, the heat resistance cannot be increased if the maximum temperature of the components does not exceed the melting temperature. However, if we assume that during melting of one of the materials, the matrix consisting of the higher-melting material remaining solid prevents the immediate removal of the melt from the electrode surface, then, for such structures, the critical temperature will be the melting temperature of the material forming the matrix or the evaporation temperature of the lower-melting material.

The heat resistance and the relative heat resistance coefficient was calculated for a number of combinations of metals with various volume contents $\varepsilon$ and 1- $\varepsilon$ by numerically solving the thermal problem (1) of heating of two-component composite materials with infinitely small sizes of the components. Temperature dependences of the volumetric heat capacity and thermal conductivity of composites and the latent heat of melting for the lower-melting material were taken into account. The results of some calculations are given in Table 3 . The upper and lower values correspond to the maximum and minimum estimates of the thermal conductivity of the composite.

\begin{tabular}{|c|c||c|c|c|c|c|}
\hline$\varepsilon$ & Re-Cu & Mo-Cu & W-Cu & Ta-Cu & W-Mo & W-Re \\
\hline \multirow{2}{*}{0.25} & 1.792 & 1.825 & 1.829 & 1.781 & 1.428 & 1.183 \\
& 1.417 & 1.675 & 1.720 & 1.504 & 1.426 & 1.126 \\
\hline \multirow{2}{*}{0.5} & 1.543 & 1.622 & 1.632 & 1.528 & 1.416 & 1.264 \\
& 1.160 & 1.456 & 1.509 & 1.237 & 1.413 & 1.182 \\
\hline \multirow{2}{*}{0.75} & 1.253 & 1.399 & 1.420 & 1.241 & 1.402 & 1.330 \\
& 0.992 & 1.288 & 1.339 & 1.047 & 1.400 & 1.263 \\
\hline
\end{tabular}

Table 3. Composite electrodes consisting of a mixture of powders

Figure 3 shows curves of $V(L)$ (Fig. $3, a$ ) and $I^{*}(L) / b$ (Fig. 3, b) for copper electrodes obtained for a inductance per unit-length of railgun channel $\lambda=0.3 \mu \mathrm{H} / \mathrm{m}$, plasmaarmature resistance $r=10^{-3} \mathrm{ohm}$, a total mass of the projectile and plasma of $1 \mathrm{~g}$, and a channel cross-section of $1 \times 1 \mathrm{~cm}$. Curves $1-3$ correspond to plasma armatures 5,10 , and 15 cm long. 

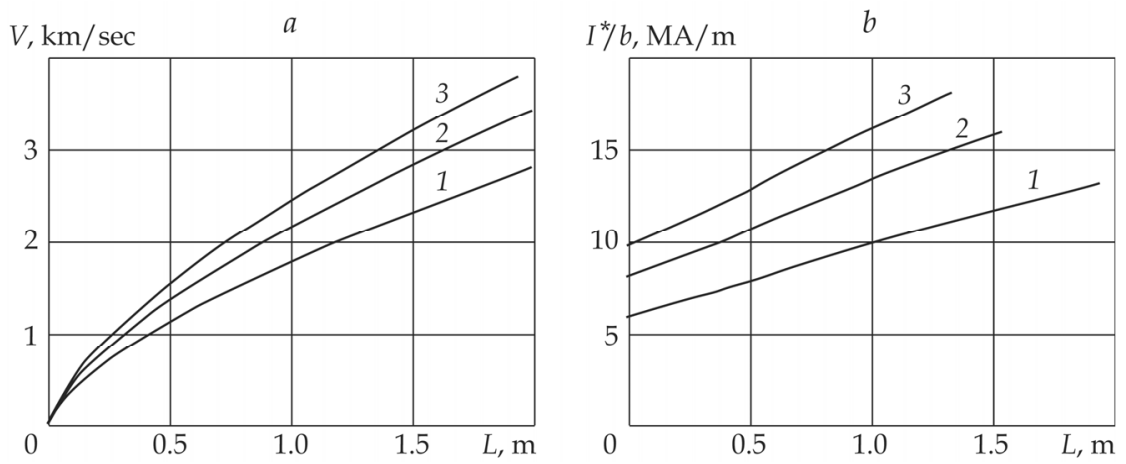

Fig. 3. Velocity $(a)$ and critical current density $(b)$ vs. plasma piston position in the railgun channel for copper electrodes. The numbers 1,2, and 3 correspond to plasma length equal $5,10,15 \mathrm{~cm}$.

Using electrodes with heat resistance twice the heat resistance of copper can lead to a factor of two increase in the critical current density and velocity, which (as seen from the figure and scale rations (4)) provides projectile velocities of 3-4 km/sec over an acceleration distance of 1 $\mathrm{m}$ and velocities of $5-7 \mathrm{~km} / \mathrm{sec}$ over an acceleration distance of $2 \mathrm{~m}$ in the regime without significant erosion of the electrodes. It can be concluded that the use of composite materials is promising for achieving high velocities in plasma-armature railgun accelerators of solids.

\section{Ultimate kinematic characteristics of conducting solids accelerated by magnetic field}

A factor limiting the attainment of high velocities during acceleration of conducting projectiles by a magnetic field is the Joule heating of conductors to temperatures above the melting point of the material. This can lead to loss of the mechanical strength of the conductors, change in their shape, and, ultimately, failure. The requirement that the conductors should not melt during acceleration imposes restrictions on the maximum permissible amplitudes of the accelerating magnetic fields, thus limiting the maximum velocity to which a conductor of given mass can be accelerated over a specified acceleration distance (Shvetsov \& Stankevich, 1992).

\subsection{Formulation}

To estimate the limits of the induction acceleration method, it is sufficient to consider the problem of the ultimate (in terms of the heating conditions) kinematic characteristics of infinite conducting flat sheets (Fig. 4) accelerated by magnetic pressure in the absence of resistance. In this section, we consider the acceleration of homogeneous sheets (Fig. 4, a), multilayer sheets (Fig. 4, b), and sheets containing a layer of composite material with electrothermal properties varying across the layer thickness (Fig. 4, c).

At the initial time $(t=0)$, the velocity of the sheet $V=0$, its temperature is $T_{0}$, and a magnetic field is absent in the sheet. In general, the electrothermal properties of the sheet (electrical conductivity $\sigma$, density $\rho$, specific heat $c$, and thermal conductivity $k$ ) can depend on the $\mathrm{x}$ coordinate of the temperature $T$. For magnetic fields typical of induction accelerators, the magnetic permeability $\mu$ of materials will be equal to the magnetic permeability of vacuum 
$\mu_{0}$. Heat transfer between the sheet and the surrounding medium and the compressibility of the sheet are neglected. We assume that the change in the internal thermal energy of the sheet is determined by Joule heating and heat transfer.
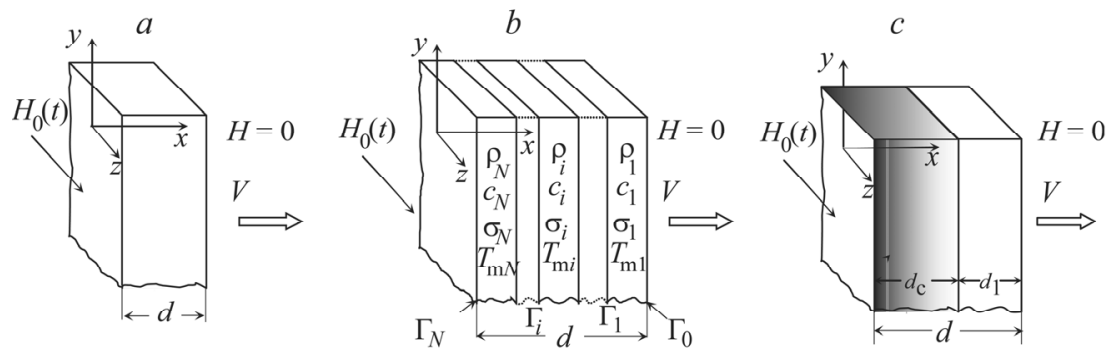

Fig. 4. Structure of accelerated sheets.

In Cartesian coordinates attached to the sheet (the boundaries of the sheet correspond to the planes $x=0$ and $x=d$ ), the distributions of the magnetic field $H(x, t)$ and temperature $T(x, t)$ in the sheet depend only on the $x$ coordinate and time $t$, and are described by the equations of magnetic field diffusion and heat conduction with the initial and boundary conditions:

$$
\begin{gathered}
\frac{\partial H}{\partial t}=\frac{1}{\mu} \frac{\partial}{\partial x}\left(\frac{1}{\sigma} \frac{\partial H}{\partial x}\right) \\
\rho c \frac{\partial T}{\partial t}=\frac{\partial}{\partial x}\left(k \frac{\partial T}{\partial x}\right)+\frac{1}{\sigma}\left(\frac{\partial H}{\partial x}\right)^{2} \\
\left.H\right|_{t=0}=0,\left.T\right|_{t=0}=T_{0},\left.H\right|_{x=0}=H_{0}(t),\left.H\right|_{x=d}=0,\left.\frac{\partial T}{\partial x}\right|_{x=0}=\left.\frac{\partial T}{\partial x}\right|_{x=d}=0 .
\end{gathered}
$$

The time dependence of the magnetic field is assumed to be known and given by the relation $H_{0}(t)=H_{\mathrm{a}} h_{0}(\tau)$, where $\tau=t / t_{0}$ ( $t_{0}$ is a characteristic time).

For sheets consisting of several layers of materials with different electrothermal properties, it is assumed that at the internal boundaries between the layers, where the properties of the medium undergo a discontinuity, the continuity of the magnetic, electrical, and thermal fields is preserved.

The velocity of the sheet $V$ and the distance $L$ traveled by it are determined by integrating the equations of motion:

$$
M \frac{d V}{d t}=\frac{\mu_{0} H_{0}^{2}(t)}{2}, \frac{d L}{d t}=V
$$

where $M=\int_{0}^{d} \rho d x$ is the mass of the sheet per unit area of its surface ( $d$ is the sheet thickness). 
The heating constraint is given by the requirement that during acceleration of the sheet at a given distance $L$, the heating of any component of the sheet materials be not higher than its melting temperature. Under this constraint and for a given function $h_{0}(\tau)$, the maximum velocity of the sheet of given structure in the general case is determined by solving the optimization problem consisting of choosing the maximum allowable (in terms of the heating conditions) values of the magnetic field amplitude $H_{\mathrm{a}}$ and the acceleration time $t_{0}$ that ensure the achievement of the maximum velocity over a given acceleration distance.

Similarity analysis for system (5) - (7) shows that for a sheet of arbitrary structure, it is sufficient to determine the maximum velocity as a function of the sheet mass $V(M, L)$ or thickness $V(d, L)$ for any one acceleration distance $L$. For any other distance $L^{\prime}$, these functions can be found using the relation:

$$
V^{\prime}\left(M^{\prime}, L^{\prime}\right)=a V(M, L), M^{\prime}=a M, V^{\prime}\left(d^{\prime}, L^{\prime}\right)=a V(d, L), d^{\prime}=a d, \quad a=\left(\frac{L^{\prime}}{L}\right)^{1 / 3}
$$

\subsection{Homogeneous sheets}

For a homogeneous "thin" sheet (in this case, the acceleration time is much longer than the time of magnetic field penetration into the sheet), direct integration of equations (5)-(7) gives (Knoepfel, 1970)

$$
V_{0}=\frac{\mu_{0} J}{2 \rho^{2}} M
$$

where $J=\int_{0}^{t} j^{2} d t=\int_{T_{0}}^{T_{\mathrm{m}}} \rho c \sigma d T$ is the current integral. In this case, the ultimate velocity of the sheet does not depend on the magnetic field pulse shape $H_{0}(t)$ and the acceleration distance and are determined only by the electrothermal properties of the sheet material and the sheet mass per unit area or the sheet thickness.

For "thick" sheets (in this case, the time of magnetic field penetration into the sheet is much greater than the acceleration time), the ultimate velocity in terms of the heating conditions can be determined from the asymptotic relation (Shvetsov \& Stankevich, 1994)

$$
V_{\infty}=\psi \sqrt{\Delta Q_{\mathrm{m}} \frac{L}{M}}
$$

where $\Delta Q_{\mathrm{m}}$ is the change in the thermal energy density of the sheet material under heating to the melting temperature and $\psi$ is a coefficient which depends on the form of the function $h_{0}(\tau)$. If the magnetic field increases monotonically with time during the acceleration, $\psi=1.1-1.2$.

Figure 5 shows the results of numerical calculations of the dependence $V(M, L)$ (curve 1) and the asymptotic dependences $V_{0}(M)$ and $V_{\infty}(M, L)$ (curves 2 and 3 for the approximations of "thin" and "thick" sheets, respectively) for a copper sheet, $L=1 \mathrm{~m}$, and a linearly increasing magnetic field. The dependence $V(M, L)$ is characterized by a velocity maximum which is reached for a certain mass or thickness of the sheet. The maximum velocity and the optimum mass depend weakly on the magnetic field pulse shape and are determined mainly by the electrothermal properties of the material and the acceleration distance. 


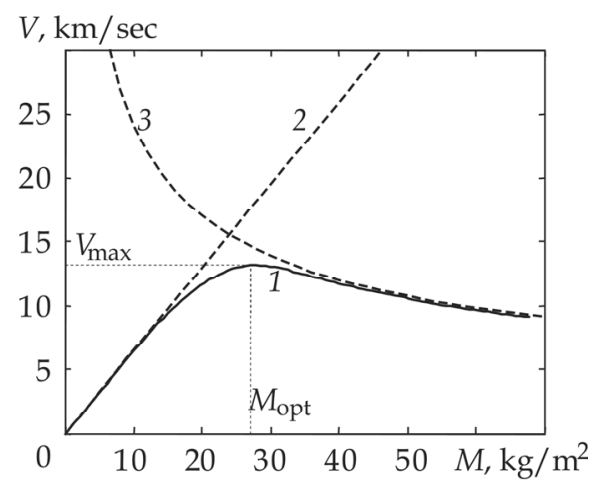

Fig. 5. Dependence $V(M)$ (curve 1 ) for $\mathrm{L}=1 \mathrm{~m}$. Curve 2 and curve 3 are asymptotic dependences obtained in the approximations of "thin" and "thick" sheets, respectively.

Figure 6, a shows curves of the ultimate velocity versus sheet mass for $\mathrm{Cu}, \mathrm{W}, \mathrm{Ti}, \mathrm{Be}, \mathrm{Fe}, \mathrm{Mo}$, $\mathrm{Ag}$, $\mathrm{Au}$, and Fig. 6, $b$ shows curves of the ultimate velocity versus sheet thickness for the same materials. It is seen that from the point of view of providing the maximum velocity, different materials can be optimal, depending on the given mass or required thickness of the sheet.

A characteristic feature of the dependences $V(M, L), V(d, L)$ (Fig. $6 a, b)$ is the presence of a velocity maximum which is reached for a certain sheet thickness $d_{\text {opt }}(L)$ or linear mass $M_{\text {otp }}(L)$ that are optimal for each material. A decrease in the maximum velocity of the sheets for $M>M_{\mathrm{opt}}$ is related to the localization of the region of maximum heating near the sheet surface, where most of the current flows because of the time of field diffusion is much greater than the time of acceleration of the sheet.
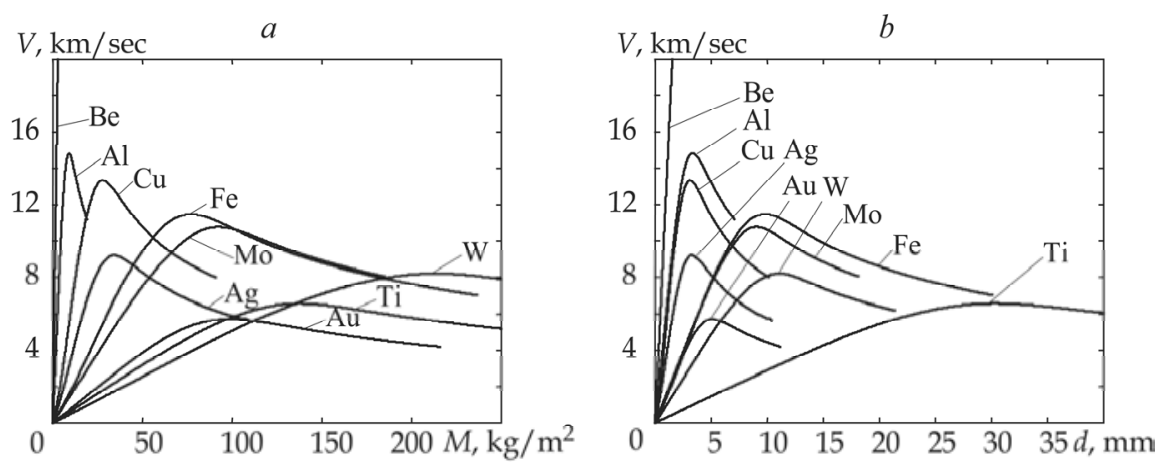

Fig. 6. Ultimate velocities vs. sheet mass (a) and vs. sheet thickness (b), for $L=1 \mathrm{~m}$

\subsection{Multilayer sheets}

In some papers (Karpova et al.,1990; Shvetsov \& Stankevich, 1992, Zaidel' , 1999), it was noted that the use of heterogeneous conductors with electrical conductivity discreet or continuously increasing with distance from the surface of the sheet can decrease their local heating considerably. 
In this subsection, we analyze the possibility of increasing the ultimate velocity of solids accelerated by a magnetic field by using multilayer conducting sheets consisting of several layers of materials with different electrothermal properties (Fig. 4, $b$ ).

A simple analytical method for optimizing the sheet structure can be obtained if we assume that the electrical properties of materials does not depend on temperature, neglect the thermal conductivity of materials, and consider steady-state solutions of equations (5) - (7) that correspond to the acceleration of sheets in an exponentially growing magnetic field $h_{0}(\tau)=e^{\tau}$.

An analysis has shown that for a given set of layer materials, the optimal structure (the sequence of layer materials and thicknesses) is the one in which the melting temperature in each layer is reached simultaneously by the time the sheet has traveled a given distance.

Solving equations (5) and (6) under the above assumptions, we obtain:

$$
\begin{gathered}
h_{i}=\sqrt{h_{i-1}^{2}-q_{i} \operatorname{cth}^{2} \lambda\left(1-\frac{q_{i-1} \sigma_{i}}{\sigma_{i-1} q_{i}}\right)}, \\
\Delta \xi_{i}=\frac{\sqrt{\sigma_{1}}}{\lambda \sqrt{\sigma_{i}}} \ln \left(\frac{h_{i}+\operatorname{cth}(\lambda) \sqrt{q_{i}}}{h_{i-1}+\operatorname{cth}(\lambda) \sqrt{q_{i-1} \sigma_{i} / \sigma_{i-1}}}\right),
\end{gathered}
$$

where $q_{i}=\rho_{i} c_{i}\left(T_{\mathrm{m} i}-T_{0}\right) / \rho_{1} c_{1}\left(T_{\mathrm{m} 1}-T_{0}\right), \Delta \xi_{i}=d_{i} / d_{1}, h_{i}$ is a dimensionless magnetic field, and $\lambda=\sqrt{t_{0} / \mu_{0} \sigma_{1} x_{1}^{2}}$ is an invariant parameter. In expressions (10) and (11), we set $h_{1}=1$, $i=2, \ldots, N$, where $N$ is the specified number of layers. In this case, as can be seen from (10), the sequence of layer materials should be chosen to reduce the values $\Delta Q_{\mathrm{m}} / \sigma$ in the direction of magnetic field diffusion.

Using the heating constraint and the equation of motion (7) and taking into account the similarity relations (8), we have:

$$
\begin{gathered}
V(\lambda)=\left(\frac{\mu_{0} \sigma_{1} L}{2}\left(\frac{\Delta Q_{\mathrm{m} 1}}{\bar{\rho} \lambda \Delta \xi \theta(\lambda)}\right)^{2}\right)^{1 / 3} \\
d(\lambda)=\left(\frac{4(\lambda \Delta \xi)^{2} L \bar{\rho} \theta(\lambda)}{\left(\mu_{0} \sigma_{1}\right)^{2} \Delta Q_{\mathrm{m} 1}}\right)^{1 / 3}
\end{gathered}
$$

where $\theta(\lambda)=\left(\operatorname{cth}(\lambda) / h_{N}\right)^{2}, h_{N}$ is the dimensionless magnetic field on the inner surface $(x=0)$ of the multilayer sheet calculated by expression (10), $\Delta \xi=1+\sum_{i=2}^{N} \Delta \xi_{i}$, and $\bar{\rho}$ is the average density of the multilayer sheet.

Figure 7 shows curves of the ultimate velocity versus linear mass of multilayer sheets calculated using analytical relations for an acceleration distance of $1 \mathrm{~m}$ (the sequence of materials is indicated in the figure).

It can be seen that the use of multilayer sheets allows a considerable increase in the ultimate velocity in terms of the heating conditions, compared to homogeneous sheets. 


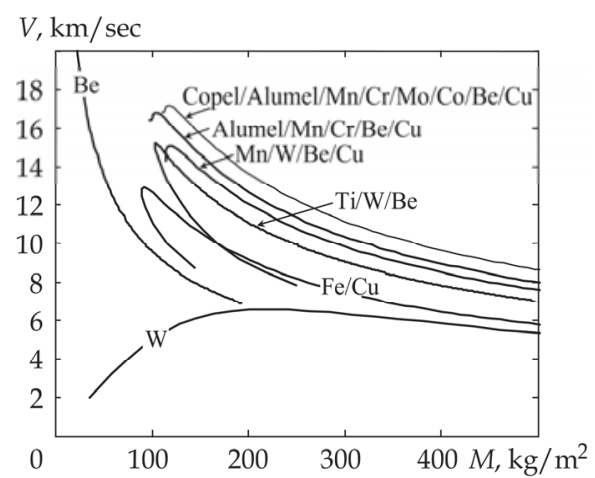

Fig. 7. Ultimate velocity versus mass of multilayer sheets for some sequences of layer material (indicated in the figure).

\subsection{Sheets with a composite layer}

Let us consider the possibility of increasing the ultimate kinematic characteristics of sheets which contain a composite material layer with electrical conductivity continuously increasing in the direction of magnetic field diffusion (Fig. 4, c).

Generally, we assume that the accelerated sheet of thickness $d$ comprises two layers in contact: a composite layer of thickness $d_{\mathrm{c}}$ consisting of a mixture of two materials (first and second) with different electrothermal properties and a homogeneous layer of thickness $d_{1}$ made of the first material (Fig. 4,c). Below, the subscripts 1 and 2 are used to denote the parameters of the first and second materials, respectively. Let the electrical conductivity of the first material be higher than the conductivity of the second material $\sigma_{1}>\sigma_{2}$, and let the electrical conductivity at different points of the composite layer be changed as a result of change in the volume concentration $\varepsilon(x)$ of the first material (the $x$ coordinate is reckoned from the sheet surface in contact with the field). Furthermore, the characteristic sizes of the particles comprising the composite are so small that it is possible to ignore the variations in the magnetic and thermal fields due to the discrete dependence of the electrothermal properties of the composite material on the coordinates. Thus, the averaged properties of the composite material are assumed to depend continuously on the $x$ coordinate according to the distribution of the volume concentration $\varepsilon(x)$ at $0 \leq x \leq d_{\mathrm{c}}$ and $\varepsilon(x)=1$ at $d_{\mathrm{c}}<x \leq d$.

The density $\rho$ and the heat capacity per unit volume $C$ for an arbitrary composite material can be obtained from the relations

$$
\begin{aligned}
& \rho(x)=\rho_{1} \varepsilon(x)+\rho_{2}(1-\varepsilon(x)), \\
& C(x)=\rho_{1} c_{1} \varepsilon(x)+\rho_{2} c_{2}(1-\varepsilon(x)) .
\end{aligned}
$$

At the same time, the dependence of the averaged electric conductivity $\sigma$ on the volume concentration $\varepsilon$ can be determined only for a composite material of known structure or experimentally. Below, we assume that the composite layer has a layered or fibrous structure (the direction of the fibers coincides with the direction of the current), then, we have

$$
\sigma(x)=\sigma_{1} \varepsilon(x)+(1-\varepsilon(x)) \sigma_{2} .
$$


The optimum distribution of the volume concentration $\varepsilon(x)$ that ensures uniform heating can be obtained in analytical form using the steady-state solutions of system (5)-(7) admissible for $h_{0}(\tau)=e^{\tau}$. The optimum law of variation of electrical conductivity in this layer can be found from the condition that the temperatures at each point of this layer reach a certain critical temperature at the end of acceleration. Using the dimensionless variables $\tilde{C}=C / C_{1}, \tilde{\sigma}=\sigma / \sigma_{1}$, and $\xi=x / d_{1}$ and the function $y(\xi)=\sqrt{\tilde{C}(\xi) / \tilde{\sigma}(\xi)}$, from (14) and (15) we obtain:

$$
\begin{gathered}
\tilde{\sigma}\left(y^{2}\right)=\frac{\tilde{C}_{2}-\tilde{\sigma}_{2}}{y^{2}\left(1-\tilde{\sigma}_{2}\right)+\tilde{C}_{2}-1} \\
\varepsilon\left(y^{2}\right)=\frac{\tilde{C}_{2}-y^{2} \tilde{\sigma}_{2}}{\tilde{C}_{2}-1+y^{2}\left(1-\tilde{\sigma}_{2}\right)}
\end{gathered}
$$

From the solution (5) and (6), for the $y(\xi)$ we obtain (Shvetsov \& Stankevich, 2003):

$$
\begin{gathered}
y^{\prime}=\frac{\mathrm{d} y}{\mathrm{~d} \xi}=\lambda \sqrt{\int_{1}^{y^{2}} \tilde{\sigma}\left(y^{2}\right) d y^{2}+\theta_{0}^{-1}(\lambda)} \\
\xi(y, y(0), \lambda)=\int_{y}^{y(0)} \frac{d y}{y^{\prime}(y, \lambda)}
\end{gathered}
$$

where $\theta_{0}(\lambda)=(\operatorname{ch} \lambda / \operatorname{sh} \lambda)^{2}$. The thickness of the composite layer $\xi_{\mathrm{c}}$ can be determined by using $y=1$ as the lower limit of integration in expression (17).

The average density of the sheet is determined from (14), (16) and (17):

$$
\bar{\rho}(y(0), \lambda)=\frac{1}{\Delta \xi} \int_{0}^{\Delta \xi} \rho \mathrm{d} \xi=\frac{1}{1+\Delta \xi_{\mathrm{c}}}\left(\int_{1}^{y_{0}} \frac{\left(\rho_{1} \varepsilon(y)+\rho_{2}(1-\varepsilon(y)) \mathrm{d} y\right.}{y^{\prime}(y, \lambda)}+\rho_{1}\right)
$$

Relations between the ultimate velocities of sheets with composite layers and the sheet mass or thickness can be derived using equations (12) and (13) in which

$$
\theta=\left(\frac{\tilde{C}_{2}-\tilde{\sigma}_{2}}{1-\tilde{\sigma}_{2}} \ln \left(\frac{y^{2}(0)\left(1-\tilde{\sigma}_{2}\right)+\tilde{C}_{2}-1}{\tilde{C}_{2}-\tilde{\sigma}_{2}}\right)+\theta_{0}^{-1}(\lambda)\right)^{-1},
$$

average density is defined by (18) and $\Delta \xi=1+\Delta \xi_{\mathrm{c}}$ is defined by (17).

Figure 8 shows curves of ultimate velocity versus sheet thickness calculated using the above analytical relations for a sheet consisting of a $\mathrm{Cu} / \mathrm{Fe}$ composite layer and a homogeneous copper layer (curves 3, 4, and 5) and curves of ultimate velocity versus thickness for homogeneous sheets of iron and copper (curves 1 and 2). For curves 4 and 5, the electrical conductivity of iron was decreased by a factor of 10 and 100, respectively.

The calculations were performed for the electrothermal properties of the materials averaged over the temperature range from room temperature to the melting point of $\mathrm{Cu}$.

It should be noted that for each value of the mass per sheet unit area $M$ or sheet thickness $d$, there is an optimal distribution $\varepsilon(x)$ that provides the attainment of the maximum velocity over a given acceleration distance. 


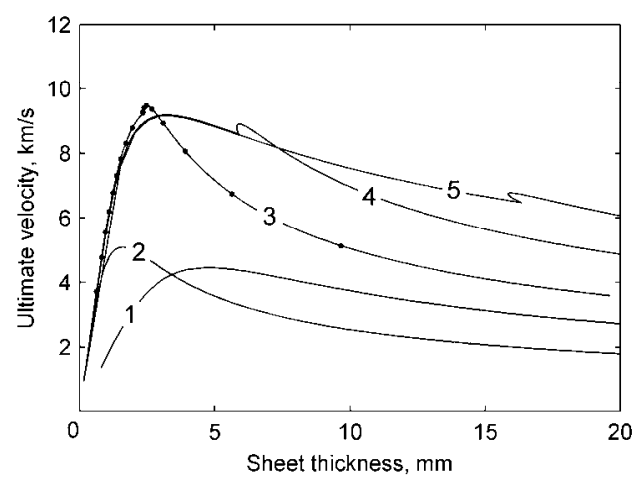

Fig. 8. Ultimate velocity versus sheet thickness for $L=0.1 \mathrm{~m}$.

As can be seen (Fig. 8), for a certain sheet thickness $d_{\text {inflection, }}$ each of curves 3,4 , and 5 has a point of inflection. The segments of the curves before the points of inflection correspond to the sheet consisting only of a composite layer. The segments of the curves behind the points of inflection were obtained for the sheet consisting of a composite layer and a homogeneous layer. On curve 3, dark circles show the points at which the volume concentration of $\mathrm{Cu}$ changes by 0.1 if $d \leq d_{\text {inflection }}$ or the relative thickness of the composite layer $v=\xi_{c} / \Delta \xi$ changes by 0.1 , if. For $d>d_{\text {inflection }}$ a certain thickness of the sheet in the neighborhood of the point of inflection, up to three different structures of the sheet that ensure its uniform heating can exist. One can see that the ultimate velocity of the sheet containing an optimized composite layer increases by a factor of about two when the total thickness of the sheet exceeds the thickness of the homogeneous copper layer for which its maximum ultimate velocity is attained.

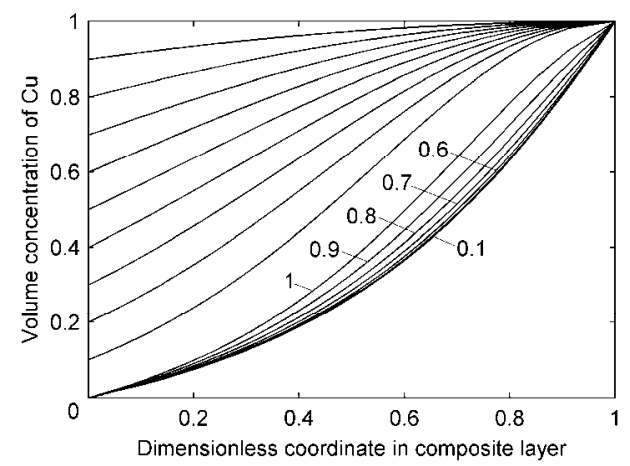

Fig. 9. Distribution of the volume concentration of $\mathrm{Cu}$ in a Fe/ $\mathrm{Cu}$ composite layers

Figure 9 shows the optimum distributions of copper concentration in the composite layer consisting of copper and iron. These distributions correspond to the points on the $\mathrm{Cu} / \mathrm{Fe}$ 
curve in Fig. 8. The curves with zero surface concentration correspond to the acceleration of the sheet consisting of a composite layer and a homogeneous copper layer. The numbers at these curves show the relative thickness of the composite layer. The curves with nonzero surface concentration of copper were obtained for a sheet consisting only of a composite layer. One can see that when the relative thicknesses of the composite layer are smaller than 0.7, the optimum profiles of copper distribution in the composite layer practically do not differ from each other.

It can be seen from Fig. 9 that as the thickness of the sheet decreases, the composite layer becomes similar in properties to a homogeneous sheet of the material with high electrical conductivity (first material). For a small values of $d$, the curve of $V(d)$ (Fig. 8) approaches the asymptote to a "thin sheet" (9) determined for the sheet of the first material. But the ultimate velocity of the composite sheet enters this asymptote for larger values of $d$ than those for the homogeneous sheet. Accordingly, its maximum ultimate velocity can be much higher than the maximum ultimate velocity of the homogeneous sheet. On the other hand, with increase in the thickness of sheets containing a composite layer of any pair of materials, the ultimate velocity always decreases, even in the case of artificial decrease in the electrical conductivity of the first material, as is the case for curves 4 and 5 in Fig. 8. However, for larger $d$, the ultimate velocity of sheets containing a composite layer is larger than the ultimate velocity of homogeneous sheets made of the materials constituting the compact. Furthermore, it is evident that with decrease in $\sigma_{2}$, the increase in ultimate velocity becomes more considerable.

The analysis performed showed that sheets containing a composite layer with electrical conductivity increasing in the direction of magnetic-field diffusion can be used to advantage to improve the ultimate (under hearting conditions) kinematic characteristics of accelerators. Thus, the ultimate velocity of a sheet containing a composite layer of $\mathrm{Fe}$ and $\mathrm{Cu}$ is about twice that of homogeneous iron and copper sheets. As the electrical conductivity of iron decreases by a factor of 100 , the ultimate velocity can increase by a factor 3 .

The analysis showed that increasing the ratio of electrical conductivities of the compact constituents, one can achieve a considerable increase in ultimate velocity compared to a homogeneous sheet. One would expect that use of conducting and nonconducting materials in combination may open up fresh opportunities. However, in this case, to ensure microuniform heating of the composite material, one would need to decrease the characteristic particle size in the compact and/or to use a material with high thermal conductivity as an insulator.

The analysis performed does not cover all aspects of the use of composite materials as current-carrying projectiles accelerated by a magnetic field. In particular, the thermomechanical and strength properties of the compact constituent materials should apparently be chosen in a special manner to ensure the integrity (nonfailure) of the projectile during acceleration.

\section{The ultimate kinematic characteristics of railguns with a metal armature}

The velocity skin effect (VSE) is a principal factor that limits the use of a metallic armature in electromagnetic railguns in the regime with sliding metallic contact (Young \& Hughes, 1982; Thornhill et al., 1989). A sharp increase in the current density due to the VSE at the contact boundary leads to fast heating of the armature in this region in excess of its melting temperature. Metallic contact is lost, and transition to the acceleration regime with plasma 
contact occurs. Some undesirable consequences may be failure of the armature, a change in its ballistic characteristics, and enhanced erosion of the rails, which reduces the life time of the EM accelerator. Furthermore, ejection of the eroded material into the interelectrode space behind the accelerated body can result in shunting of the current and deterioration in acceleration. As the ultimate velocity for the regime with sliding metallic contact, Long and Weldon (Long \& Weldon, 1989) proposed to consider as an ultimate velocity for sliding metallic contact such a value of the velocity $V$ so that the metallic armature can be accelerated providing that its maximum temperature does not exceed its melt point. For traditional homogeneous materials, the ultimate velocity is usually lower than $1 \mathrm{~km} / \mathrm{sec}$ (Long \& Weldon, 1989; Shvetsov \& Stankevich, 1992), and this currently limits the use of conducting solids in railguns.

A considerable number of papers deal with the search for methods of increasing the critical (ultimate) velocity, or, in other words, decreasing the current concentration due to the VSE.

This subsection is concerned with analyzing the ultimate velocity versus projectile mass at fixed acceleration distance for various methods of decreasing the current density at the railarmature interface. The analysis is performed by numerical solution of the system of equations of unsteady magnetic-field diffusion and unsteady heat transfer in a two dimensional formulation. Homogeneous and multilayer projectiles and homogeneous rails and rails with a resistive coating are considered.
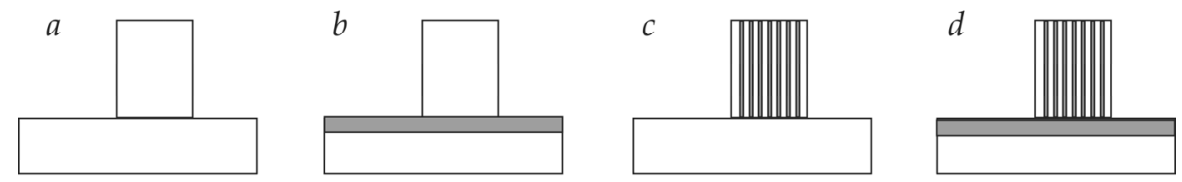

Fig. 10. Configuration of calculation regions. Homogeneous armature and rails $(a)$, homogeneous armature and rails with a resistive layer $(b)$, multilayer armature and homogeneous rails $(c)$, and multilayer armature and rails with a resistive layer $(d)$.

\subsection{Formulation of the problem}

We consider the acceleration of homogeneous and multilayer conducting solids in electromagnetic launchers with homogeneous rails and rails with a high resistive layer (Fig.10). Accelerated bodies will be called armature or projectile, as is common in the literature. The time-dependent distributions of the magnetic field and the temperature of the armature and rails were determined by numerical solution of the system of unsteady equations of magnetic-field diffusion and heat transfer in a two-dimensional formulation. Neglecting the effects associated with the system finiteness in the direction $z$ and displacement currents, these equations in a moving frame of reference connected with the armature can be written in the form:

$$
\begin{gathered}
\mu_{0}\left(\frac{\partial H}{\partial t}-V \frac{\partial H}{\partial x}\right)=\frac{\partial}{\partial x} \frac{1}{\sigma} \frac{\partial H}{\partial x}+\frac{\partial}{\partial y} \frac{1}{\sigma} \frac{\partial H}{\partial y} \\
\rho c\left(\frac{\partial T}{\partial t}-V \frac{\partial T}{\partial x}\right)=\frac{\partial}{\partial x} k \frac{\partial T}{\partial x}+\frac{\partial}{\partial y} k \frac{\partial T}{\partial y}+\frac{1}{\sigma}\left(\frac{\partial H}{\partial x}\right)^{2}+\frac{1}{\sigma}\left(\frac{\partial H}{\partial y}\right)^{2} .
\end{gathered}
$$


It was assumed that the electrothermal properties of materials do not depend on temperature and there is ideal electric and thermal contact on the boundaries between the armature and the rails and between the resistive coating and the support, the continuity conditions for the magnetic field, temperature, the tangential component of the electric field, and the normal components of the current density and heat flux are satisfied. The magnetic field in the railgun channel was assumed to be known and equal to the linear current density through the armature.

The velocity of the armature $V$ and the distance $L$ traveled by it are determined by integrating the equation of motion:

$$
M \frac{d V}{d t}=\frac{\lambda}{2}\left(\frac{I}{b}\right)^{2}, \frac{d L}{d t}=V
$$

\subsection{Homogeneous armature and resistive layer}

The effect of a resistive layer on the ultimate kinematic characteristics of a homogeneous armature was studied in a series of calculations for three materials of the layer with considerably different electrical conductivities: titanium $\left(\sigma=1.8 \cdot 10^{6}(\mathrm{Ohm} \cdot \mathrm{m})^{-1}\right)$, Copel alloy (Ni: $42.5-44 \%, \mathrm{Mn}: 0.1-0.5 \%$, Cu: the rest, $\left.\sigma=0.21 \cdot 10^{6}(\mathrm{Ohm} \cdot \mathrm{m})^{-1}\right)$, and graphite $\left(\sigma=0.04 \cdot 10^{6}(\mathrm{Ohm} \cdot \mathrm{m})^{-1}\right)$. The thickness of resistive layer $d$ was $0 \div 1.2 \mathrm{~mm}$. Armatures made of aluminum, copper, and tungsten were examined. The acceleration distance was $1 \mathrm{~m}$.

Use of a resistive layer has an ambiguous effect on the rate of change in the maximum armature temperature, and hence, and the ultimate velocity. The ultimate velocity can both increase and decrease, depending on the thickness and conductivity of the layer, the electrothermal properties and dimensions of the armature, and the specified acceleration distance.

Two regimes are typical of heating in the armature. In the first of this, the change in the maximum temperature in the armature is primarily determined by Joule heating of the armature, and the second regime occurs when the armature is heated as a result of increase in the temperature of the contact boundaries due to Joule heating of the resistive layer.

The increase in the maximum temperature in the armature due to Joule heating of the resistive layer proceeds mainly in the initial stage of acceleration. As the armature is accelerated, the heating of the resistive layer decreases, and so does the maximum temperature of the armature. With a further increase in the velocity, the Joule heating of the armature due to current concentration caused by the velocity skin effect becomes more intense, and the maximum temperature begins to increase again.

Figure $11 a, b$ shows the dependences of the ultimate velocity on the thickness of the resistive layer for a aluminum armature (Fig. 11,a), copper armature (Fig. 11, b) with various lengths in the direction of motion and for various materials of coatings calculated for an acceleration distance of $1 \mathrm{~m}$. The figures on the curves denote the lengths of armatures in $\mathrm{mm}$. The continuous curves refer to the resistive coating of graphite, the dotted curves to titanium and the dashed curves to Copel.

For all the armature materials studied, use of resistive coatings of titanium and Copel leads to an increase in the ultimate velocity compared to the case where rails without coating are used. A titanium layer ensures an only $15 \% \div 20 \%$ increase in the velocity for acceleration of an $\mathrm{Al}$ armature (Fig.11, a). The dependences for $\mathrm{W}$ and $\mathrm{Cu}$ armatures accelerated on rails with a titanium coating show the same relative increment of the velocity as for an $\mathrm{Al}$ 
armature. Unlike a titanium coating, a Copel layer increases the ultimate velocity by a factor of 2 or 3 . As can be seen from Fig. 11, the ultimate velocity decreases with increase in the length of the armature. This dependence of the ultimate velocity of the length of the armature is typical of cases where Joule heating of the armature, whose intensity is determined by the current concentration due to the VSE, plays a predominant role.
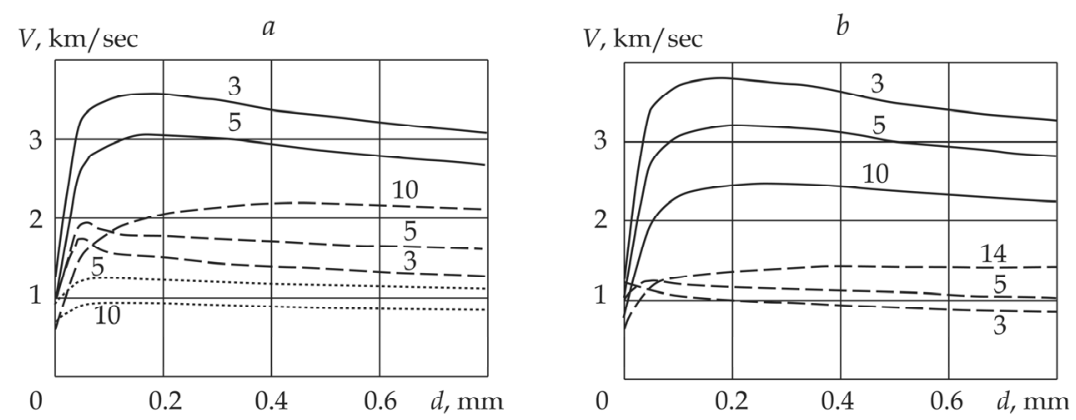

Fig. 11. The dependences of the ultimate velocity on the thickness of the resistive layer for a plane aluminum armature $(a)$ and copper armature $(b)$.
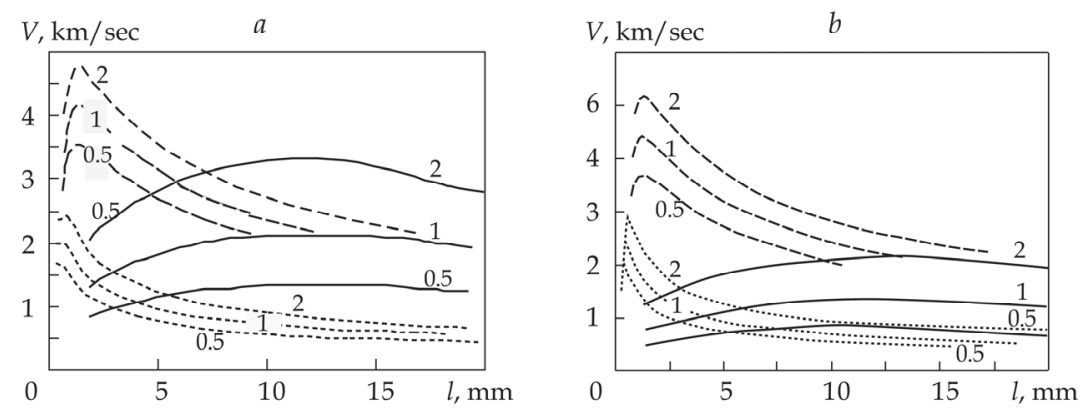

Fig. 12. The dependences of the ultimate velocity on the length of an armature.

Figures $12 a, b$ give dependences of the ultimate velocity on the length of an armature calculated at various acceleration distances $L=0.5 \mathrm{~m}, 1 \mathrm{~m}$, and $2 \mathrm{~m}$ for homogeneous armatures of $\mathrm{Al}(a)$ and $\mathrm{Cu}(b)$. The dotted curves in these figures show the dependences obtained for the copper rails without a resistive layer. The dashed curves show the dependences obtained using a Copel resistive layer, and the solid curves correspond to a graphite resistive layer. The figures on the curves denote the acceleration distance $(\mathrm{m})$.

It is seen that a reasonable choice of the coating material can lead to a significant (severalfold) increase in the ultimate velocity.

\subsection{Multilayer armature and homogeneous rails}

Let us consider the ultimate kinematic characteristics of multilayer armatures with orthotropic conductivity (4.3.1) and multilayer armatures with alternating layers of materials with high and low conductivity (4.3.2) during acceleration in railgun. 


\subsubsection{Multilayer armature with insulating layers}

An increase in the ultimate velocity in terms of the heating conditions can be achieved through the use of a multilayer armature consisting of a set of alternating conducting and insulating layers (Fig. 10, c) (Shvetsov \& Stankevich, 1997). The insulating layers provide rapid penetration of the field from the middle part of the armature to the interface, due to the infinite rate of field diffusion in these layers. This increases the size of the region of current flow through the interface, thus reducing the current density and armature heating rate. This effect is the greater the smaller the thickness of the layers and the larger the distance between the rails $h$.

To illustrate the potential of this type of armatures, we consider an armature with orthotropic conductivity, assuming infinitely small sizes of the insulating and conducting layers. Using Faraday's law and taking into account that at the center of symmetry of the armature (for $y=h / 2$ ), the $x$ component of the electric field $E_{x}=0$ and that the tangential component of the electric field is continuous on the contact boundary, we obtain the equation describing the distribution magnetic field in the armature (Shneerson et al., 1996):

$$
\frac{\partial H}{\partial t}=\frac{1}{\mu \sigma_{\text {armature }}} \frac{\partial^{2} H}{\partial x^{2}}+\left.\frac{2}{\mu \sigma_{\text {rail }} h} \frac{\partial H}{\partial y}\right|_{y=0}
$$

The effect of the singularities on the contact boundary due to both the relative motion of the conductors and the passage of the current around the corner point is described by the last term on the right side of this equation, which can be made arbitrarily small by choosing a sufficiently large value of $h$.

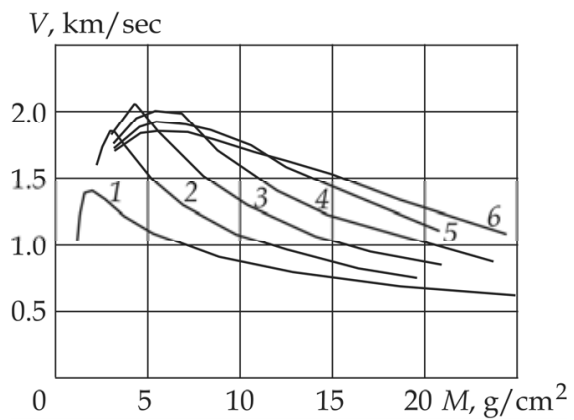

Fig. 13. Multilayer tungsten armature. Curves 1 to 6 correspond to numbers of layers $1,4,6$, 10,16 , and 22, respectively.

Figure 13 shows the ultimate velocity of a tungsten armature versus its mass for various numbers of tungsten layers with insulating layers between them. The bore cross-section is $b \times h=2 \mathrm{~cm} \times 2 \mathrm{~cm}$, and the acceleration distance is $1 \mathrm{~m}$. The curves labeled 1 to 6 correspond to numbers of layers of $1,4,6,10,16$, and 22 .

One can see a shift of the maximum toward large masses and a total increase in the velocity for large masses.

Figures 14 give dependences $V(d)$ obtained for armatures composed of conducting layers of aluminum (Fig. 14, a) and tungsten (Fig. 14, b). Curves 1, 2, and 3 correspond to a multilayer armature $(N=22)$ with $h=b=1,2$, and $4 \mathrm{~cm}$, respectively; curve 4 corresponds to a 
homogeneous armature; and curve 5 to induction acceleration of the same multilayer armature for which the equations of motion (21) are valid. Indexes a, b, c correspond to acceleration distances $0.5 \mathrm{~m}, 1 \mathrm{~m}$, and $2 \mathrm{~m}$ respectively. The ultimate velocity of the multilayer armature in the railgun can be seen to be much higher than that of the homogeneous armature; however, for the given range of $h$ values, it remains much lower (about two times) than the velocity achieved during induction acceleration.
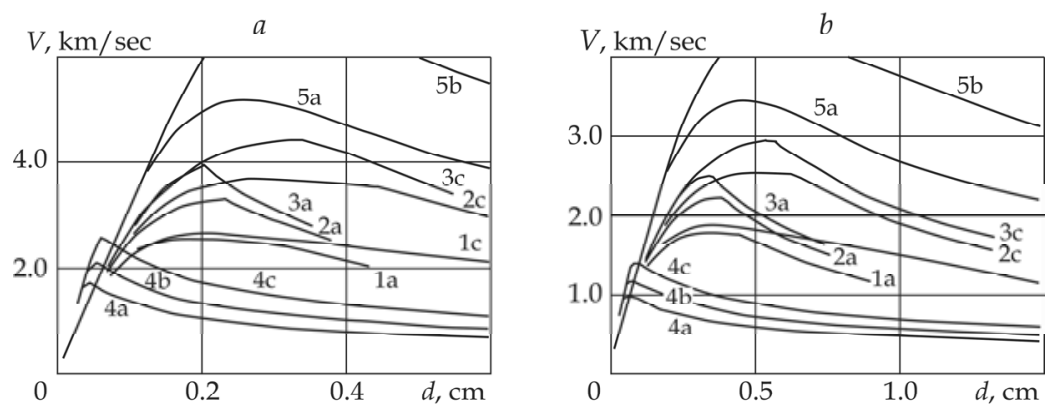

Fig. 14. The dpendances of the ultimate velocity for aluminum (a) armatures and tungsten (b) multilayer armatures.

A comparison was made of the ultimate kinematic characteristics for two projectile configurations: a multilayer armature 2 with orthotropic electrical conductivity and a nonconducting armature of stabilized structure 3 (Fig. 15, a, b).
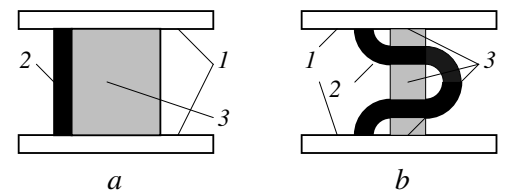

Fig. 15. (a) plane multilayer armature, (b) shaped multilayer armature. 1 rails; 2 armature; 3 supporting structure.

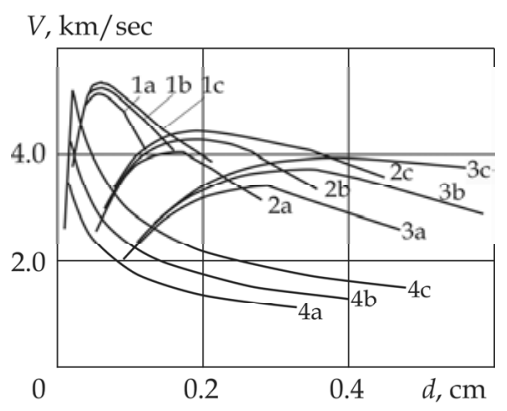

Fig. 16. Shaped aluminum armature. Curves 1 to 3 correspond to $f=1,0.5,0.3$. Curves 4 are for homogeneous armature. 
Let us consider some ultimate velocities of a multilayer armature of mass $M_{\mathrm{a}}$ which has a nonconducting supporting structure of mass $M_{\mathrm{s}}$ to provide stability of its motion. Suppose that the ratio $f=M_{\mathrm{a}} / M$ remains constant with a change in the total mass $M=M_{\mathrm{a}}+M_{\mathrm{s}}$. Figure 16 shows dependences $V(M)$ for an armature with conducting tungsten layers $(N=22)$. Curves 1,2 , and 3 correspond to values $f=1,0.5$, and 0.3 , respectively; curves 4 correspond to a homogeneous flat armature; letters $\mathrm{a}, \mathrm{b}$, and c denote acceleration distances $L=0.5,1$, and $2 \mathrm{~m}$, respectively. One can see that in the range of large masses, the ultimate velocity of the shaped armature far exceeds the ultimate velocity of the flat armature.

\subsubsection{Multilayer armature with high and low electroconductivity layers}

Figure 17 presents dependences $V(M)$ for an armature composed of copper and titanium alloy layers $\left(\sigma=1.8 \cdot 10^{6}(\Omega \cdot \mathrm{m})^{-1}\right)$. The curves labeled $1,2,3$, and 4 , correspond to values of $N=1,4,6,10$. One can see that the maximum value of the ultimate velocity of this armature exceeds the ultimate velocity of the homogeneous copper armature, but the increase in the velocity achieved for this armature is smaller than that for the multilayer armature with insulating layers.

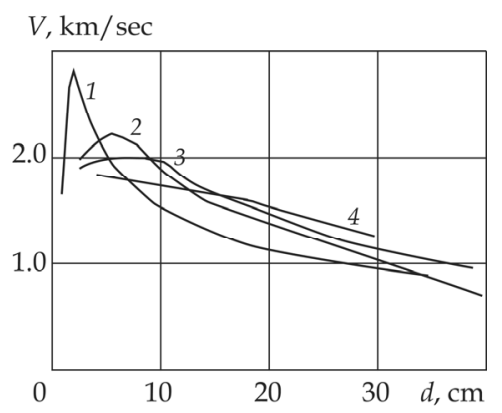

Fig. 17. Copper and titanium alloy composed armature. The curves 2 to 4 correspond to $N=4,6,10$.

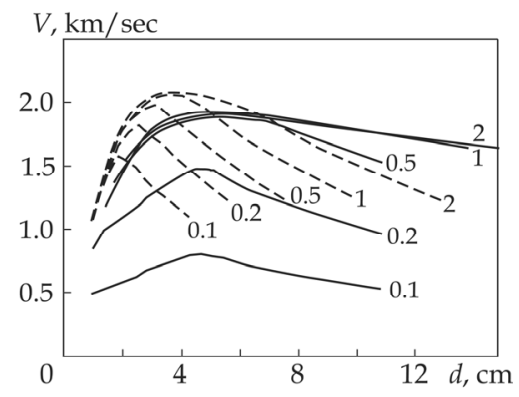

Fig. 18. Ultimate velocity as a function of the multilayer tungsten armature length for acceleration distances of $0.1,0.2,0.5,1$, and $2 \mathrm{~m}$. 


\subsection{Multilayer armature and rails with resistive layer}

In railguns with homogeneous rails, the ultimate velocities of armatures consisting of alternating conducting and nonconducting layers, far exceed the ultimate velocities of homogeneous armatures (see Fig. 13 and 14). In addition, for armatures with orthotropic conductivity, the maximum ultimate velocity is reached for greater armature lengths compared to homogeneous armatures.

The curves of the ultimate velocities of a multilayer tungsten armature versus its length $l$ calculated for acceleration distances of $0.1,0.2,0.5,1$, and $2 \mathrm{~m}$ are given in Fig. 18. The dotted curves show the calculation results for a multilayer armature and rails without a coating (see Fig. 10, c), and the solid curves show the results for the same armature and rails with a resistive Copel alloy coating (see Fig. 10,d). The figures on the curves indicate the acceleration distances in meters. It is evident that, indeed, there is a shift of the maximum ultimate velocity toward larger values of $l$, and this maxima is rather flat.

It is important that in this case, the ultimate velocity does not depend on the acceleration distance for $L>0.5 \mathrm{~m}$. The above is true for $\mathrm{Al}$ and $\mathrm{Cu}$ armatures with orthotropic conductivity, and for $L \geq 0.5 \mathrm{~m}$. This indicates that the dimensions of the region of the contact boundary through which the current passes are determined mainly by the properties and dimensions of the resistive coating (in this case, Copel alloy).

The results obtained indicate that a resistive coating can be used to advantage to decrease the current concentration in the armature due to the velocity skin effect. This considerably decelerates armature heating near the contact boundaries. As a result, the ultimate velocity to which the armature can be accelerated in a channel of a specified length with retention of solid metal contact with the rails can be increased by a factor of $2 \div 4$ and the kinetic energy of the armature can be increased by a factor of $4 \div 16$ compared to the case of rails without a coating. With a further decrease in the conductivity of the resistive layer, the current concentration in the armature decreases, but, in this case, overheating and failure of the resistive layer can take place.

The ultimate maximum velocity of a multilayer armature increases with an increase in the number of layers and bore height $h$. For $h=2 \div 6 \mathrm{~cm}$ and an acceleration distance of $2 \mathrm{~m}$, the ultimate velocity can reach $2 \div 4 \mathrm{~km} / \mathrm{sec}$ for multilayer copper and aluminum armatures and $1.8 \div 2.8 \mathrm{~km} / \mathrm{sec}$ for multilayer tungsten armature. These ultimate velocities are $2 \div 3.5$ times higher those of a homogeneous armature having the same mass.

Thus, the analysis shows that the ultimate velocities attained for homogeneous materials can be considerably increased by changing the structure and thermophysical properties of projectile and rail materials.

It should be noted that the use of resistive coatings in a number of features that can considerably lower the attainable velocities. First, the velocity of magnetic-field diffusion along a resistive coating is higher than the diffusion velocity in the armature. As a result, the armature current flows along the contact boundary in the opposite direction to the rail current. Interaction of these currents gives rise to a magnetic-pressure force that repels the contact surfaces. If special precautions are not taken, this can lead to a loss of metal contact between the projectile and the rails. Since the repelling force decreases as the magnetic field penetrates into the armature and the armature velocity increases, one method for overcoming this problem is to use a resistive coating with conductivity decreasing in a predetermined manner in the direction of motion. Second, the resistive layer fails under the considerable thermal stresses caused by sharp temperature variations on the boundaries of 
the resistive layer. These stresses can be reduced using a resistive material with high thermal conductivity, melting point, and mechanical strength.

\section{Comparison between $2 \mathrm{D}$ and $3 \mathrm{D}$ electromagnetic modeling of railgun}

The results presented in fourth subsection have shown that the ultimate (with respect to heating conditions) kinematic characteristics of launchers depend greatly on the electrothermal properties and the structure of the materials used, the projectile weight, the acceleration dynamics determined by the shape of the current pulse, and the acceleration distances. They can be considerably increased (severalfold) by using multilayer and composite conductors as current-carrying elements of launchers and by optimizing the current pulse shape.

However, these results were obtained by numerical modeling of launchers in a twodimensional spatial formulation. Thus, it is unclear how the maximum current density on the contact boundary, its dependent armature heating rate, and the ultimate kinematic characteristics in a real launcher differ from the values obtained by two-dimensional modeling. The purpose of this section is to compare results of 2-D and 3-D calculations of armature heating for various armature shapes, matched acceleration dynamics, and totalcurrent distribution curves.

\subsection{Formulation of the problem}

Comprehensive three-dimensional modeling of a rail launcher requires considerable computational resources; therefore, in the present work, we confine ourselves to a consideration of the launcher region which includes the armature and part of the rails in immediate proximity to the armature (Fig. 19). The symmetry of the problem allows the model to be simplifies to one fourth of the armature and rails. This area is of greatest interest because it is in the neighborhood of the armature that the distribution of the magnetic field and current has a substantially three-dimensional form.

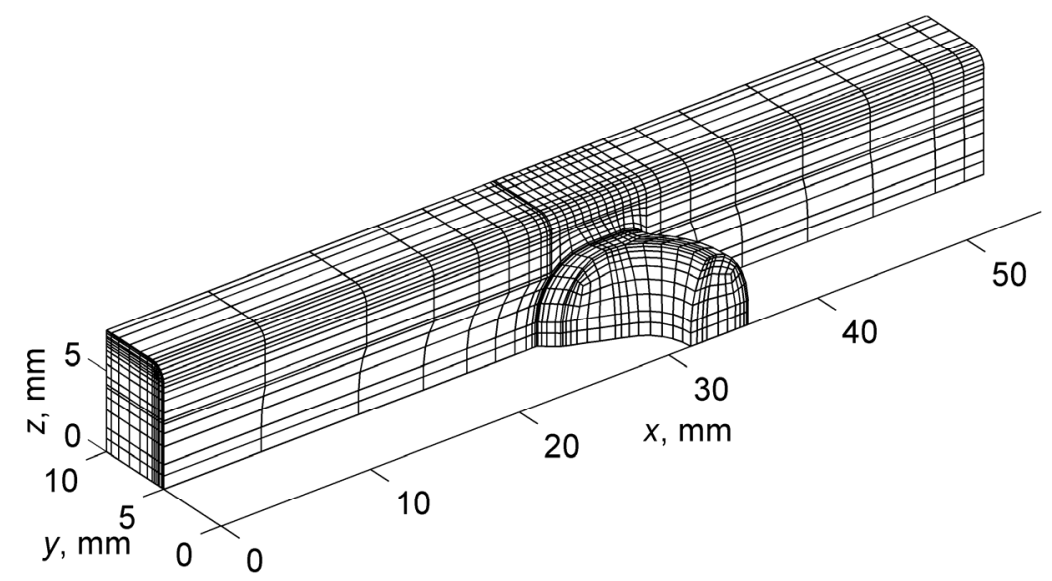

Fig. 19. Model of an electromagnetic railgun. 
Further simplification of the problem can be achieved by taking into account that, even at relatively low velocities of armature motion $(\sim 50 \mathrm{~m} / \mathrm{sec})$, the currents flow primarily in a thin surface layer on the armature and their distribution is determined mainly by the shape of the armature and rails and by the velocity of the armature. In this area, the current density distribution can found using steady-state solutions of the Maxwell equations. In addition, we assume that, during acceleration, ideal electrical and thermal contact between the armature and rails is preserved and the electrothermal properties of the materials remain constant (do not depend on temperature).

From Maxwell equations we obtain a system of equations for the vector and scalar potentials $(A, \varphi)$, which, for the region of conductors (armature and rails), in a moving frame of reference connected with the armature, can be written as follows:

$$
\begin{gathered}
j=-\frac{1}{\mu_{0}} \nabla^{2} \boldsymbol{A}=-\sigma(\nabla \varphi-\boldsymbol{V} \times \nabla \times \boldsymbol{A}) \\
\nabla \cdot j=\nabla \cdot(\sigma(\nabla \varphi-V \times \nabla \times A))=0
\end{gathered}
$$

In the surrounding nonconducting space, where the current density, $\mathbf{j}=0$ instead of (22) we have:

$$
\nabla^{2} \boldsymbol{A}=0
$$

The temperature distribution in the armature and rails is given by the solution of the unsteady heat-conduction equation:

$$
\rho c\left(\frac{\partial T}{\partial t}+\boldsymbol{V} \cdot \nabla T\right)-\nabla \cdot k \nabla T=\frac{j^{2}}{\sigma}
$$

The electromagnetic part of the problem, in fact, contains one boundary condition, which requires that the components of the vector potential vanish at an infinite boundary. At all inner boundaries between the conductors and the surrounding nonconducting space, the continuity conditions for the vector-potential components and their derivatives are satisfied.

The boundary conditions for the heat-conduction equation (25) specify the absence of heat transfer with the surrounding medium during acceleration and reduce to Neumann conditions at all boundaries of the conductors.

\subsection{Numerical solution procedure}

Equations (22), (23), (25) were approximated by the Galerkin weighted residual finiteelement method in the conductors, and the boundary element method was used outside the conductors. The numerical technique is described in more detail in (Shvetsov \& Stankevich, 2009).

Owing to the stationary character of the electromagnetic part of the posed problem, the solutions for the electromagnetic and thermal problems can be obtained separately. At the first stage, for a certain set of armature velocities $V_{i}\left(i=1, \ldots N, V_{i}=|\mathbf{V}|\right)$ and the total current in the launcher $I$, we solved problem (22)-(24) and found the distributions of the current 
density in the armature and rails $\mathbf{j}\left(\mathbf{r}, V_{i}\right)$ and the accelerating magnetic pressure forces $F\left(V_{i}\right)$. The times $t_{i}$ (that correspond to the velocities $V_{i}$ ) were calculated by integrating the equation of motion $M \mathrm{~d} V / \mathrm{d} t=F(V)$, where $M$ is the total mass of the accelerated projectile and $F(V)$ the interpolated dependence of the accelerating magnetic force. After that, we solved the armature heating problem using the time dependence of the current density interpolated over the known values of $\mathbf{j}\left(\mathbf{r}, t_{i}\right)$ in the right side of Eq. (25) (Shvetsov \& Stankevich, 2011).

To compare 3-D and 2-D modeling results, in addition to 3D modeling, we solved the problem of armature and rail launcher heating in a 2-D formulation (19), (20). The computation domain had the geometry of the middle section of a 3-D launcher (plane $z=0$ in Fig. 19). The value of the magnetic field in the launcher channel was set equal to the linear current density $I / b$ ( $b$ is the rail width). In the two-dimensional formulation, the time dependence of the armature velocity obtained in the 3-D calculations was used. For a comparison with the 3-D modeling results, we solved the two-dimensional problem in two steps: first calculated the current density distributions as a function of the armature velocity and then solved the unsteady thermal problem.

\subsection{Results}

In the first step, we calculated the current density distributions and magnetic field, the accelerating magnetic force, and the inductance per unit length for an electromagnetic rail launcher with a $1 \times 1 \mathrm{~cm}$ channel cross-section, copper rails $1.5 \mathrm{~cm}$ wide and $0.5 \mathrm{~cm}$ thick (Fig. 19). To study the effect of the armature shape and its electrothermal properties on the current density distribution, heating dynamics, and inductance gradient, we considered various armature shapes: rectangular, standard C-shaped, cylindrical, modified C-shaped, saddle-shaped (Figs. $20 a, b, c, d, e$, respectively) made of copper and an $\mathrm{Al}$ alloy.
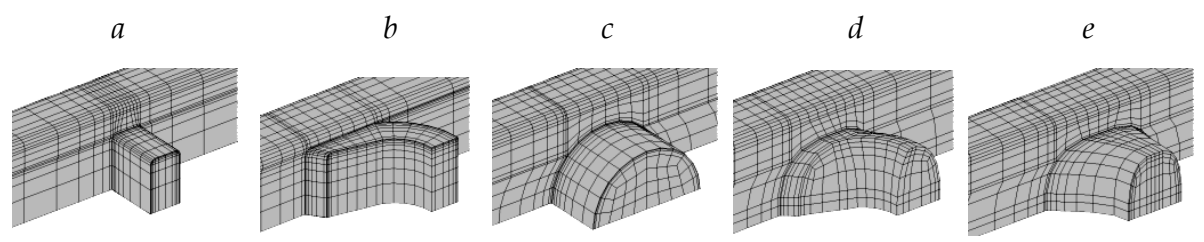

Fig. 20. Armature shapes. a) rectangular, $b$ ) standard C shaped, $c$ ) cylindrical, $d$ ) curved C shaped, e) saddle-shaped.

The calculations show that the maximum current density is reached on the perimeter of the rail-armature interface. This results, first, from the presence of the geometrical singularity at the armature-rail interface and, second, from the velocity skin-effect, which pushes the current toward the rear boundary of the perimeter of the contact interface. Here it should be noted that the presence of the edges formed by the intersection of the flat lateral surfaces of the armature favors an additional increase in the current density at the points of intersection of the edges with the rail surface (Satapathy et al., 2007). The position of the point at which the maximum absolute value of the current density is reached depends on the shape, electrical conductivity, and velocity of the armature. This point can be located on the frontal, rear or any other part of the perimeter. We note that, 
regardless of the material for rectangular and cylindrical armatures, the current density maximum is always located on the rear part of the interface. As the armature velocity increases, the velocity skin effect begins to play a dominant role and the current density maximum, which may initially be located on the frontal part of the curve, is shifted along the perimeter of the interface to its rear part. This process was observed for all the remaining armature shapes studied. The armature velocity, at which this shift begins, depends on the electrical conductivity of the armature. For armatures of identical shape, the lower the electrical conductivity of the armature, the higher the velocity at which the shift of the current density maximum begins.

In two-dimensional modeling of the armature current density distribution under the adopted boundary conditions, the maximum current density is always reached at the point of contact of the rear boundary of the armature to the rail boundary. Thus, the 2D model does not allow the current density distribution to be calculated correctly for armatures for which the maximum current density is reached (in a certain velocity range) on the frontal part of the contact boundary, in particular, for C-shaped armatures. However, by modifying the shape of such an armature by rounding its leg and the frontal side (Fig. 20, c), it is possible to achieve a more uniform current density distribution along the perimeter of the contact interface with transition of the current density maximum to rear boundary at velocities of $100 \mathrm{~m} / \mathrm{sec}$ for the copper armature and $200 \mathrm{~m} / \mathrm{sec}$ for the aluminum armature.

In the second step, the technique described above was used to calculate the nonstationary temperature distribution in the armature. It was assumed that the armature is accelerated in a rail accelerator at a constant total current equal to $100 \mathrm{kA}$.
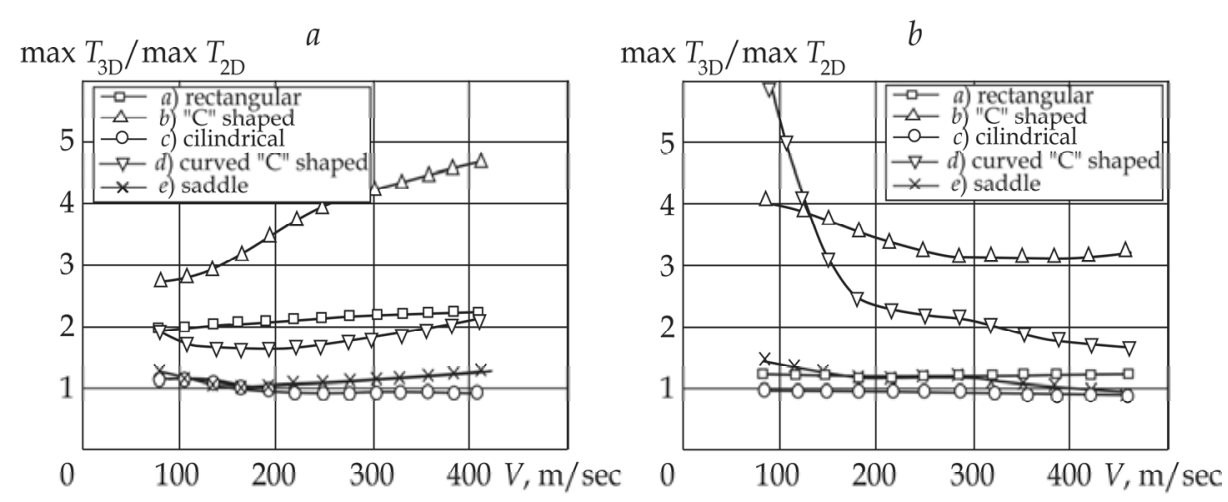

Fig. 21. Ratio of the maximum armature temperatures obtained by $3 \mathrm{D}$ and $2 \mathrm{D}$ calculations versus $\mathrm{Cu}(a)$ and $\mathrm{Al}(b)$ armature velocity

The ratio of the maximum armature temperatures obtained by the $3 \mathrm{D}$ and $2 \mathrm{D}$ calculations $\left(\vartheta(V)=\max T_{3 \mathrm{D}} / \max T_{2 \mathrm{D}}\right)$ for armatures of various shapes versus velocity are given for copper armatures in Fig. 21, $a$ and for armatures made of aluminum alloy in Fig. 21, $b$. The mass of copper armatures of various shapes was a) $2.66 \mathrm{~g}, b) 3.54 \mathrm{~g}, c) 6.95 \mathrm{~g}$, d) $4.13 \mathrm{~g}$, e) $5.43 \mathrm{~g}$. The mass for armatures of aluminum alloy a) $0.82 \mathrm{~g}$, b) $1.08 \mathrm{~g}, c) 2.13 \mathrm{~g}$, d) $1.27 \mathrm{~g}$, and e) $1.67 \mathrm{~g}$. 
It is evident that for almost all armature shapes (except for the copper cylindrical armature) $\vartheta(V)$ is larger than unity. The ratio of the maximum armature temperatures obtained in the 3-D and 2-D calculation depends weakly on the velocity, especially at high armature velocities. The value of this ratio is determined primarily by the armature shape. A significant difference between the $3 \mathrm{D}$ and $2 \mathrm{D}$ calculation results for the maximum temperature is observed for the $\mathrm{C}$ shaped (Fig. 21). This is due to the fact that, in these cases, the region of the maximum heating at low velocities is located on the frontal contact interface, which is not described by two-dimensional modeling. For the cylindrical and saddle-shapes armatures, the temperatures ratio $\vartheta(V)$ is close to unity.

From the results it follows that, for armatures of some geometrical shapes (in our case, these are cylindrical and saddle shapes), a two-dimensional description of the armature heating is in reasonable agreement with that in a three-dimensional description. Hence, one can hope that the severalfold increase in the ultimate (under heating conditions) kinematic characteristics predicted by the two-dimensional modeling may be achieved for real rail accelerators of solid bodies.

\section{Conclusion}

A study was made of the effect of the structure and electrothermal properties of the materials of the electrodes and projectiles on the ultimate kinematic characteristics of three types of electromagnetic launchers: plasma armature railguns, coilguns, and railguns with conducting bodies. Homogeneous, layered, and composite materials were considered. The study shows that by controlling the structure and electrothermal properties of electrode materials and projectiles, it is possible to significantly reduce the thermal constraints and increase the ultimate projectile velocities by a factor of two to four.

\section{References}

Barber J. P. (1972). The acceleration of macroparticles and a hypervelocity electromagnetic accelerator, Ph.D. Thesis, The Australian National University.

Barber J. P., Bauer D. P, Jamison K., Parker J. V., Stefani F. \& Zielinski A. A. (2003). Survey of armature transition mechanisms, IEEE Transaction on Magnetics, Vol. 39, No. 1, part 1. (January,2003), pp. 47-51, ISSN 0018-9464.

Fair H. D. (2005). Electromagnetic Launch Science and Technology in the United States Enters a New Era, IEEE Transaction on Magnetics, Vol. 41, No. 1, (January, 2005), pp. 158-164, ISSN 0018-9464.

Fair H. D. (2007). Progress in Electromagnetic Launch Science and Technology, IEEE Transaction on Magnetics, Vol. 43, No. 1, (January, 2007), pp. 93-98, ISSN 00189464.

Jackson G. L., Farria L. K. \& Tower M. M. (1986). Electromagnetic railgun extended-life bore material test results, IEEE Transaction on Magnetics, Vol. 22, No. 6, (December 1986), pp. 1542-1545, ISSN 0018-9464.

Harding J. T., Kaplan R. B., Pierson H. O., Tuffias R. H. \& Upshaw J. L. (1986). Chemically vapor deposited materials for railguns, IEEE Transaction on Magnetics, Vol. MAG22, No. 6, (December 1986), pp. 1506-1509, ISSN 0018-9464. 
Haugh D. C. \& Gilbert S. (2003). U. K. Electric Gun National Overview, IEEE Transaction on Magnetics, Vol. 39, No.1, (January, 2003), pp. 18-21, ISSN 0018-9464.

Hawke R. S. \& Scudder J. K. (1979). Magnetic propulsion railguns: their design and capabilities. Proceedings of 2nd International Conference on Megagauss Magnetic Fields Generation and Related Topics, Plenum Press, New York and London, ISBN 0-30640461-3, Washington, D.C. May 30-June 1, 1979, pp. 297-311.

Karpova I. M., Semakhin A. N., Titkov V. V. \& Shneerson G. A. (1990). Analysis of Methods of Lowering Heating of and Thermal Stresses in the Coils in High pulsed Magnetic Fields, Proceedings of 5th International Conference on Megagauss Magnetic Fields Generation and Related Topics, Nova science Publisher, New York, ISBN 0-941743-861, Novosibirsk, USSR, July 3 - 7, 1989.

Knoepfel H. (1970) Pulsed High Magnetic Fields, North Holland, ISBN 0471885320, Amsterdam - London.

Lehmann P. (2003). Overview Of the Electric Launch Activities at the French-German Institute of Saint-Louis (ISL), IEEE Transaction on Magnetics, Vol. 39, No.1, (January, 2003), pp. 24-28, ISSN 0018-9464.

Long G.C., Weldon W.F. (1989). Limits to the velocity of solid armature in railgun, IEEE Transaction on Magnetics, Vol. 25, No. 1, (January, 1989), pp. 347-352, ISSN 00189464.

Powell J. D. (1984). Therma1-energy transfer from arc to rails in arc-driven railgun, IEEE Transaction on Magnetics, Vol. MAG-20, No. 2, (February, 1984), pp. 395-398, ISSN 0018-9464.

Satapathy S., Watt T \& Persad Ch. (2007) Effect of Geometry Change on Armature Behavior, IEEE Transaction on Magnetics, 2007. Vol. 43, No. 1. (January, 2007), pp. 408-412, ISSN 0018-9464.

Shneerson G. A., Karpova I. M. \& Titkov V. V. (1996). The Inverse Problem of Superfast Contact Theory for an Accelerated Body with Orthotropic Conduction, Journal of Applied Mechanics and Technical Physics, Vol. 37, No. 2, pp. 200-205, ISSN 0021-8944.

Shrader J. E., Bohn A. J. \& Thompson J. G. (1986). Railgun experimental results due to varying bore and arc materia1s, and varying the number of barrel turns, IEEE Transaction on Magnetics, Vol. MAG-22, No. 6, (November 1986), pp. 1739-1741, ISSN 0018-9464.

Shvetsov G. A., Anisimov A. G., Bashkatov Yu. L. \& Chistyakov V. P. (1987) Railgun accelerators of macroparticles, Part 1: General characteristics. Proceedings of 4 th International Conference on Megagauss Magnetic Fields Generation and Related Topics, Plenum Press, New York, 1987, ISBN 0-306-42574-2, Santa Fe, New Mexico, USA, July 14-17, 1986, pp. 775-794, ISBN 0-306-42574-2.

Shvetsov G. A., Anisimov G. A., Stankevich S. V. et al. (1992). Interaction between plasma piston and railgun electrodes, Proceedings of 8th IEEE Internationa1 Pulsed Power Conference, IEEE Publishing Service, 1992, ISBN 0-7803-0176-5, San Diego, CA., June 16-19, 1991, pp. 771-777.

Shvetsov G. A. \& Stankevich S. V. (1992). Ultimate Velocities of Magnetically Driven Plates, Proceedings of 6th International Conference on Megagauss Magnetic Fields Generation and 
Related Topics, Nova Science Publisher Inc., 1992, ISBN 1-56072-160-X, Albuquerque, New Mexico, Nov. 8-11, 1992. pp. 852-857.

Shvetsov G. A. \& Stankevich S. V. (1994). Ultimate Velocities of Plates Accelerated by Magnetic Field, Journal of Applied Mechanics and Technical Physics, Vol. 35, No. 3, (May, 1994), pp. 336-344, ISSN 0021-8944.

Shvetsov G. A. \& Stankevich S. V. (1995). Critical current density in railguns with composite electrodes, IEEE Transaction on Magnetics, Vol. 31, No. 1, (January 1995), pp. 237242, ISSN 0018-9464.

Shvetsov G. A. \& Stankevich S. V. (1997). Ultimate kinematic characteristics of armatures with ortotropic and anisotropic electroconductivity, IEEE Transaction on Magnetics, Vol. 33, No. 1, (January 1997), pp. 266-271, ISSN 0018-9464.

Shvetsov G. A., Rutberg P. G. \& Kolikov V. A. (2001). Problems, Results and Prospects of Electric Launch in Russia, IEEE Transaction on Magnetics, Vol. 37, No.1, part I of two part, (January, 2001), pp. 42-45, ISSN 0018-9464.

Shvetsov G. A., Rutberg P. G. \& Savvateev A. F. (2003). Results of Resent Research on Electromagnetic Launch Technology in Russia, IEEE Transaction on Magnetics, Vol. 39, No.1, (January, 2003), pp. 29-34, ISSN 0018-9464.

Shvetsov G. A. \& Stankevich S. V. (2003). Ultimate Kinematic Characteristics of Composite Solids, IEEE Transaction on Magnetics, Vol. 39, No.1, (January, 2003), pp. 327-331, ISSN 0018-9464.

Shvetsov G. A., Rutberg P. G. \& Budin A. V. (2007). Overview of Some Resent Research in Russia, IEEE Transaction on Magnetics, Vol. 43, No.1, (January, 2007), pp. 99-106, ISSN 0018-9464.

Shvetsov G. A. \& Stankevich S. V. (2009). Effect of Shaper of Metal Solids on their Joule Hearting in Electromagnetic Rail Launchers, Journal of Applied Mechanics and Technical Physics, Vol. 50, No. 2, (Mach, 2009), pp. 342-351, ISSN 0021-8944.

Shvetsov G. A. \& Stankevich S. V. (2011). Three-dimensional numerical modeling of the joule hearting of various shapes of armatures in railgun, IEEE Transaction on Plasma Science, Vol. 39, No. 1, Part I of two parts, (January, 2011), pp. 456-460, ISSN 00933813.

Thornhill L. D., Batteh J. D. \& Brown J. L. (1989). Armature Options for Hypervelocity Railguns, IEEE Transaction on Magnetics, Vol. 25, No. 1, (January, 1989), pp. 552-557, ISSN 0018-9464.

Wang Y., Cheng S. \& Zheng P. (2003). Widely Developing Electric Launch Technology in China, IEEE Transaction on Magnetics, Vol. 39, No.1, (January, 2003), pp. 39-41, ISSN 0018-9464.

Vrable D. L, Rosenwasser S. N. \& Korican J. A. (1991). Design and fabrication of an advanced, lightweight, high stiffness, railgun barrel concept, IEEE Trans. on Magnetics, Vol. 27, No. 1, (January, 1991), pp. 470-475, ISSN 0018-9464.

Young F. J. \& Hughes W.F., (1982). Rail and armature current distribution in electromagnetic launchers, IEEE Transaction on Magnetics, Vol. 18, No 1, (January,1982), pp. 33-41, ISSN 0018-9464. 
Zaidel' R. M. (1999). Composite electrodynamic liner, Journal of Applied Mechanics and Technical Physics, Vol. 40, No. 5, (September, 1999), pp. 777-783, ISSN 00218944. 


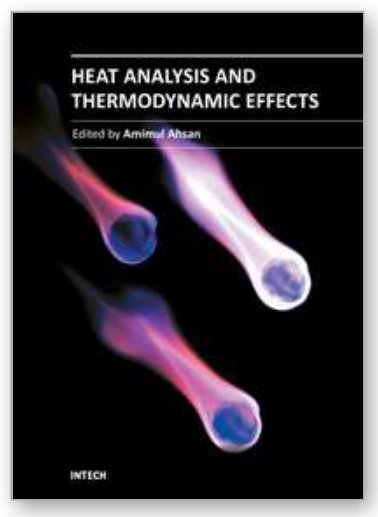

\author{
Heat Analysis and Thermodynamic Effects \\ Edited by Dr. Amimul Ahsan
}

ISBN 978-953-307-585-3

Hard cover, 394 pages

Publisher InTech

Published online 22, September, 2011

Published in print edition September, 2011

The heat transfer and analysis on heat pipe and exchanger, and thermal stress are significant issues in a design of wide range of industrial processes and devices. This book includes 17 advanced and revised contributions, and it covers mainly (1) thermodynamic effects and thermal stress, (2) heat pipe and exchanger, (3) gas flow and oxidation, and (4) heat analysis. The first section introduces spontaneous heat flow, thermodynamic effect of groundwater, stress on vertical cylindrical vessel, transient temperature fields, principles of thermoelectric conversion, and transformer performances. The second section covers thermosyphon heat pipe, shell and tube heat exchangers, heat transfer in bundles of transversely-finned tubes, fired heaters for petroleum refineries, and heat exchangers of irreversible power cycles. The third section includes gas flow over a cylinder, gas-solid flow applications, oxidation exposure, effects of buoyancy, and application of energy and thermal performance index on energy efficiency. The forth section presents integral transform and green function methods, micro capillary pumped loop, influence of polyisobutylene additions, synthesis of novel materials, and materials for electromagnetic launchers. The advanced ideas and information described here will be fruitful for the readers to find a sustainable solution in an industrialized society.

\title{
How to reference
}

In order to correctly reference this scholarly work, feel free to copy and paste the following:

Gennady Shvetsov and Sergey Stankevich (2011). Problem of Materials for Electromagnetic Launchers, Heat Analysis and Thermodynamic Effects, Dr. Amimul Ahsan (Ed.), ISBN: 978-953-307-585-3, InTech, Available from: http://www.intechopen.com/books/heat-analysis-and-thermodynamic-effects/problem-of-materials-forelectromagnetic-launchers

\section{INTECH}

open science | open minds

\author{
InTech Europe \\ University Campus STeP Ri \\ Slavka Krautzeka 83/A \\ 51000 Rijeka, Croatia \\ Phone: +385 (51) 770447 \\ Fax: +385 (51) 686166 \\ www.intechopen.com
}

\author{
InTech China \\ Unit 405, Office Block, Hotel Equatorial Shanghai \\ No.65, Yan An Road (West), Shanghai, 200040, China \\ 中国上海市延安西路65号上海国际贵都大饭店办公楼 405 单元 \\ Phone: +86-21-62489820 \\ Fax: +86-21-62489821
}


(C) 2011 The Author(s). Licensee IntechOpen. This chapter is distributed under the terms of the Creative Commons Attribution-NonCommercialShareAlike-3.0 License, which permits use, distribution and reproduction for non-commercial purposes, provided the original is properly cited and derivative works building on this content are distributed under the same license. 